\title{
A generalized mass involving higher order symmetric function of the curvature tensor
}

\author{
YanYan $\mathrm{Li}^{*}$ and Luc Nguyen ${ }^{\dagger}$
}

January 22, 2018

\begin{abstract}
We define a generalized mass for asymptotically flat manifolds using some higher order symmetric function of the curvature tensor. This mass is nonnegative when the manifold is locally conformally flat and the $\sigma_{k}$ curvature vanishes at infinity. In addition, with the above assumptions, if the mass is zero, then, near infinity, the manifold is isometric to a Euclidean end.
\end{abstract}

\section{Introduction}

Let $(M, g)$ be an $n$-dimensional Riemannian manifold. Assume that $(M, g)$ is asymptotically flat, i.e. there is a compact set $K \subset M$, some $R \geq 1$ and a diffeomorphism $\Phi: M \backslash K \rightarrow \mathbb{R}^{n} \backslash B_{R}$ such that

$$
\left(\Phi^{*} g\right)_{i j}(x)=\delta_{i j}+o\left(|x|^{-\tau}\right) \text { as }|x| \rightarrow \infty .
$$

If $\tau \geq \frac{n-2}{2}$ and the scalar curvature $R_{g}$ is integrable, then the so-called ADM mass of $(M, g)$ is defined by (see [1, 2])

$$
m=\int_{S_{\infty}}\left(g_{i j, j}-g_{j j, i}\right) d S^{i},
$$

where $g_{i j, k}$ denotes a partial derivative and $d S^{i}$ is the normal surface element of $S_{\infty}$, the sphere at infinity.

\footnotetext{
*Mathematics Department, Rutgers University. Partially supported by NSF Grant DMS-1203961

${ }^{\dagger}$ Mathematics Department, Princeton University
} 
That $m$ is a geometric invariant of $(M, g)$ is a consequence of the following expansion of the Hilbert-Einstein action:

$$
R_{g} * 1=d\left(g^{a b} \omega_{a}^{c} \wedge \eta_{c b}\right)+g^{a b} \omega_{d}^{c} \wedge \omega_{a}^{d} \wedge \eta_{b c}
$$

where $R_{g}$ is the scalar curvature, $* 1$ is the volume form, $\omega_{a}{ }^{b}$ is the Levi-Civita connection one-form with respect to a frame $\left\{e_{a}\right\}$ and $\left.\eta_{a b}=\left(e_{a} \wedge e_{b}\right)\right\rfloor * 1$. See Bartnik [2] for more details.

Mass and its properties have attracted much attention since it was introduced. One of the reason is its wide range of applications in mathematical relativity and in geometric analysis. For example, consider the Yamabe problem which asks to find on a compact Riemannian manifold a conformal metric with constant scalar curvature. Its solutions are critical point of the Hilbert-Einstein functional in a fixed conformal class. Thus, it is not too surprising that the notion mass is useful in the study of the Yamabe problem. In fact, it is very important in the solution of the Yamabe problem [21] as well as in the resolution of compactness issue of the Yamabe problem [24, 19, 8, 17, 18, 20, 13].

In recent years, fully nonlinear versions of the Yamabe problem have received much attention after the work of Viaclovsky [28, 29, 30] and of Chang, Gursky and Yang [3, 4, 15, 6]; see e.g. [11, 14, 15, 9, 12, 25, 26, 16, 27]. For a metric $g$, let $A_{g}$ be the Schouten tensor of $g$, i.e.

$$
A_{g}=\frac{1}{n-2}\left(\operatorname{Ric}_{g}-\frac{1}{2(n-1)} R_{g} g\right)
$$

where $\operatorname{Ric}_{g}$ and $R_{g}$ denote the Ricci curvature and the scalar curvature of $g$. Let $\lambda\left(A_{g}\right)=\left(\lambda_{1}, \cdots, \lambda_{n}\right)$ denote the eigenvalues of $A_{g}$ with respect to $g$. For $1 \leq k \leq n$, let $\sigma_{k}(\lambda)=\sum_{1 \leq i_{1}<\cdots<i_{k} \leq n} \lambda_{i_{1}} \cdots \lambda_{i_{k}}, \lambda=\left(\lambda_{1}, \cdots, \lambda_{n}\right) \in \mathbb{R}^{n}$, denote the $k$-th elementary symmetric function, and let $\Gamma_{k}$ denote the connected component of $\{\lambda \in$ $\left.\mathbb{R}^{n} \mid \sigma_{k}(\lambda)>0\right\}$ containing the positive cone $\left\{\lambda \in \mathbb{R}^{n} \mid \lambda_{1}, \cdots, \lambda_{n}>0\right\}$.

Question 1.1 Let $(N, h)$ be a compact, smooth Riemannian manifold of dimension $n \geq 3$ satisfying $\lambda\left(A_{h}\right) \in \Gamma$ on $N$ and $1 \leq k \leq n$. Is there a smooth positive function $u$ on $N$ such that $\hat{h}=u^{\frac{4}{n-2}} h$ satisfies

$$
\sigma_{k}\left(\lambda\left(A_{\hat{h}}\right)\right)=1, \quad \lambda\left(A_{\hat{g}}\right) \in \Gamma_{k}, \quad \text { on } N ?
$$

Equation (24) is a second order fully nonlinear elliptic equation of $u$. The special case of Question 1.1 for $k=1$ is the Yamabe problem in the so-called positive case.

The problem is in general not a variational one when $k \geq 3$. Natural variants of equation (2) which are of variational form have been introduced by Chang and Fang 
[7]; see also a subsequent paper of Graham [10] on the algebraic structure of these equations under conformal transformations.

From the discussion on the relation between the ADM mass and the Yamabe problem, it is natural to ask if there is some notion of mass associated with the $\sigma_{k}$ Yamabe problem. The main goal of this note is to give some generalization along this line. While we have not been able to identify such a notion that is directly related to the $\sigma_{k}$ curvature, we are able to do so for a variant for $2 \leq k<\frac{n}{2}$, which coincides with the $\sigma_{k}$ curvature when $(M, g)$ is locally conformally flat. This is motivated by a relation between the Pffafian and the $\sigma_{n / 2}$ curvature (see Viaclovsky [28]). See Theorem 3.1 for a precise definition.

After our work was done, the Yanyan Li heard a talk by Guofang Wang in the conference on 'Geometric PDEs' in the trimester on 'Conformal and Kähler Geometry' at IHP in which he announced that he together with Yuxin Ge and Jie Wu had also developed a notion of higher order mass. After the talk, Yanyan informed Guofang that we had defined a mass by using an invariant of the $\sigma_{k}$ curvature which agrees with the $\sigma_{k}$ curvature when the manifold is locally conformally flat and proved that the mass is non-negative under the assumption that the manifold is locally conformally flat and the $\sigma_{k}$ curvature is zero near infinity, together with a rigidity in that case.

The rest of the paper is organized as follows. In Section 2, we define a curvature invariant $\Lambda_{k}$ which coincides with the $\sigma_{k}$ curvature when $(M, g)$ is locally conformally flat. We also provide a decomposition for $\Lambda_{k}$ which is a generalization of the decomposition (11) for the Hilbert-Einstein action. In Section 3, we used the decomposition developed in Section 2 to define a mass, called the $k$-th mass. In Section 4 , we announce a very restrictive version of the positive mass theorem for the $k$-th mass.

Acknowledgment. The authors would like to thank Professor Deser for his remarks which help improve the presentation of the paper.

\section{A curvature invariant}

Consider an $n$-dimensional Riemannian manifold $(M, g)$. Let $\left\{e_{1}, \ldots, e_{n}\right\}$ be a local orthonormal frame and $\left\{\theta^{1}, \ldots, \theta^{n}\right\}$ its dual coframe. Let $\left(\omega_{i}^{j}\right)$ be the skew-symmetric matrix of Levi-Civita connection one-forms:

$$
\nabla_{\xi} e_{i}=\omega_{i}^{j}(\xi) e_{j}
$$

(Here and below, upper indices label rows while lower indices label columns.) The first structural equations read

$$
d \theta^{j}=\theta^{i} \wedge \omega_{i}^{j}
$$


The curvature tensor is viewed as a skew-symmetric matrix of two-forms,

$$
\Omega_{i}{ }^{j}(X, Y)=\theta^{j}\left(R(X, Y) e_{i}\right)
$$

The second structural equations read

$$
\Omega_{i}^{j}=d \omega_{i}^{j}-\omega_{i}^{k} \wedge \omega_{k}^{j}
$$

Also, the first and second Bianchi identities read

$$
\theta^{i} \wedge \Omega_{i}{ }^{j}=0 \text { and } d \Omega_{i}{ }^{j}=-\Omega_{i}{ }^{k} \wedge \omega_{k}{ }^{j}+\omega_{i}{ }^{k} \wedge \Omega_{k}{ }^{j} .
$$

For a multi-index $I=\left(i_{1}, \ldots, i_{m}\right)$, let $\theta^{I}=\theta^{i_{1}} \wedge \ldots \wedge \theta^{i_{m}}$ and $\theta^{[I]}=* \theta^{I}$ where $*$ is the Hodge dual operator.

For $1 \leq k \leq \frac{n}{2}$, define the $n$-form

$$
\Lambda_{k}=\frac{1}{2^{k} k !} \sum_{I=\left(i_{1}, \ldots, i_{2 k}\right) \in S_{n, 2 k}} \Omega_{i_{1}}^{i_{2}} \wedge \ldots \wedge \Omega_{i_{2 k-1}}^{i_{2 k}} \wedge \theta^{[I]}
$$

where

$$
S_{n, 2 k}:=\left\{\left(i_{1}, \ldots, i_{2 k}\right): 1 \leq i_{p} \leq n, i_{p} \neq i_{q} \text { whenever } p \neq q\right\} .
$$

It is useful to observe that

$$
\Lambda_{k}=\frac{1}{2^{k} k !} \sum_{1 \leq i_{1}, \ldots, i_{2 k} \leq n} \Omega_{i_{1}}^{i_{2}} \wedge \ldots \wedge \Omega_{i_{2 k-1}}^{i_{2 k}} \wedge \theta^{[I]} .
$$

For $k=1, * \Lambda_{1}$ is half the scalar curvature. For $n$ even and $k=n / 2, \Lambda_{n / 2}$ is the Pfaffian. Those quantities are frame independent. We claim that this is true for all $\Lambda_{k}$ 's. Indeed, let $P$ be an orthonormal matrix function, $\tilde{e}_{i}=P_{i}{ }^{j} e_{j}, \tilde{\theta}^{j}=\left(P^{-1}\right)_{i}{ }^{j} \theta^{i}$. We have

$$
\tilde{\omega}_{i}^{j}=P_{i}^{k} \omega_{k}^{l}\left(P^{-1}\right)_{l}^{j}+P_{i}^{k} d\left(P^{-1}\right)_{k}^{j} \text { and } \tilde{\Omega}_{i}^{j}=P_{i}^{k} \Omega_{k}^{l}\left(P^{-1}\right)_{l}^{j} .
$$


We then have

$$
\begin{aligned}
& 2^{k} k ! \tilde{\Lambda}_{k}=\sum_{1 \leq i_{1}, \ldots, i_{2 k} \leq n} \tilde{\Omega}_{i_{1}}^{i_{2}} \wedge \ldots \wedge \tilde{\Omega}_{i_{2 k-1}}^{i_{2 k}} \wedge \tilde{\theta}^{[I]} \\
& =\sum_{1 \leq i_{1}, \ldots, i_{2 k} \leq n}\left[P_{i_{1}}^{p_{1}}\left(P^{-1}\right)_{p_{2}}{ }^{i_{2}} \Omega_{p_{1}}{ }^{p_{2}}\right] \wedge \ldots \wedge\left[P_{i_{2 k-1}}^{p_{2 k-1}}\left(P^{-1}\right)_{p_{2 k}}^{i_{2 k}} \Omega_{p_{2 k-1}} p_{2 k}\right] \\
& \wedge *\left(\left[\left(P^{-1}\right)_{q_{1}}^{i_{1}} \theta^{q_{1}}\right] \wedge \ldots \wedge\left[\left(P^{-1}\right)_{q_{2 k}}{ }^{i_{2 k}} \theta^{q_{2 k}}\right]\right) \\
& =\sum_{1 \leq i_{1}, \ldots, i_{2 k} \leq n} P_{i_{1}}^{p_{1}}\left(P^{-1}\right)_{q_{1}}^{i_{1}}\left(P^{-1}\right)_{p_{2}}{ }^{i_{2}}\left(P^{-1}\right)_{q_{2}}{ }^{i_{2}} \ldots \\
& \ldots P_{i_{2 k-1}}^{-1} p_{2 k-1}\left(P^{-1}\right)_{q_{2 k-1}} i_{2 k-1}\left(P^{-1}\right)_{p_{2 k}} i_{2 k}\left(P^{-1}\right)_{q_{2 k}}{ }^{i_{2 k}} \\
& \Omega_{p_{1}}^{p_{2}} \wedge \ldots \wedge \Omega_{p_{2 k-1}}^{p_{2 k}} \wedge *\left(\theta^{q_{1}} \wedge \ldots \wedge \theta^{q_{2 k}}\right) \\
& =\sum_{1 \leq p_{1}, \ldots, p_{2 k} \leq n} \Omega_{p_{1}}^{p_{2}} \wedge \ldots \wedge \Omega_{p_{2 k-1}}^{p_{2 k}} \wedge *\left(\theta^{p_{1}} \wedge \ldots \wedge \theta^{p_{2 k}}\right) \\
& =2^{k} k ! \Lambda_{k},
\end{aligned}
$$

where in the second-to-last identity we have used the orthogonality of $P$.

When $g$ is conformally flat, $* \Lambda_{k}$ is proportional to the $\sigma_{k}$-curvature. This was noticed by Viaclovsky [28] in case $k=n / 2$. The argument for general $k$ is similar. We include it here for completeness. Recall that the Riemann curvature tensor Riem admits the decomposition

$$
\text { Riem }=W_{g}+A_{g} \odot g
$$

where $\odot$ is the Kulkarni-Nomizu product and $W_{g}$ is the Weyl tensor of $g$. When $(M, g)$ is locally conformally flat, $W_{g} \equiv 0$. Fix a point $p \in M$. The local orthonormal frame $\left\{e_{1}, \ldots, e_{n}\right\}$ is chosen so that $A_{g}$ is diagonalized at $p$ with eigenvalue $\lambda_{1}, \ldots, \lambda_{n}$; in particular, $A_{i}{ }^{j}=\lambda_{i} \delta_{i j}$. We have at $p$ that

$$
\begin{aligned}
\Omega_{i}{ }^{j} & =\operatorname{Riem}_{i}{ }_{k l} \theta^{k} \wedge \theta^{l} \\
& =\left(A_{g} \odot g\right)_{i}{ }^{j}{ }_{k l} \theta^{k} \wedge \theta^{l} \\
& =\left(A_{i k} \delta_{j l}-A_{j k} \delta_{i l}+A_{j l} \delta_{i k}-A_{i l} \delta_{j k}\right) \theta^{k} \wedge \theta^{l} \\
& =2\left(\lambda_{i}+\lambda_{j}\right) \theta^{i} \wedge \theta^{j} .
\end{aligned}
$$


It follows that, also at $p$,

$$
\begin{aligned}
\Lambda_{k} & =\frac{1}{2^{k} k !} \sum_{I=\left(i_{1}, \ldots, i_{2 k}\right) \in S_{n, 2 k}} \Omega_{i_{1}}^{i_{2}} \wedge \ldots \wedge \Omega_{i_{2 k-1}} i_{2 k-2} \wedge \theta^{[I]} \\
& =\frac{1}{k !} \sum_{I=\left(i_{1}, \ldots, i_{2 k}\right) \in S_{n, 2 k}}\left(\lambda_{i_{1}}+\lambda_{i_{2}}\right) \ldots\left(\lambda_{i_{2 k-1}}+i_{2 k}\right) \underbrace{\theta^{I} \wedge \theta^{[I]}}_{=d v_{g}} \\
& =\frac{1}{k !} \sum_{I=\left(i_{1}, \ldots, i_{2 k}\right) \in S_{n, 2 k}}\left(\lambda_{i_{1}}+\lambda_{i_{2}}\right) \ldots\left(\lambda_{i_{2 k-1}}+i_{2 k}\right) d v_{g} \\
& =\frac{2^{k}(n-k) !}{k !(n-2 k) !} \sum_{J=\left(j_{1}, \ldots, j_{k}\right) \in S_{n, k}} \lambda_{j_{1}} \ldots \lambda_{j_{k}} d v_{g} \\
& =\frac{2^{k}(n-k) !}{(n-2 k) !} \sigma_{k}\left(A_{g}\right) d v_{g},
\end{aligned}
$$

i.e. $\Lambda_{k}$ is proportional to $\sigma_{k}\left(A_{g}\right) d v_{g}$.

To finish this section, we derive a decomposition of $\Lambda_{k}$ which we will need later. By the second structural equations,

$$
\begin{aligned}
2^{k} k ! \Lambda_{k}= & \sum_{1 \leq i_{1}, \ldots, i_{2 k} \leq n} \Omega_{i_{1}}{ }^{i_{2}} \wedge \ldots \wedge \Omega_{i_{2 k-3}}{ }^{i_{2 k-2}} \wedge d \omega_{i_{2 k-1}}{ }^{i_{2 k}} \wedge \theta^{[I]} \\
& -\sum_{1 \leq i_{1}, \ldots, i_{2 k} \leq n} d \Omega_{i_{1}}{ }^{i_{2}} \wedge \ldots \wedge \Omega_{i_{2 k-3}}{ }^{i_{2 k-2}} \wedge \omega_{i_{2 k-1}}{ }^{p} \wedge \omega_{p_{k}}{ }^{i_{2 k}} \wedge \theta^{[I]} \\
= & \sum_{1 \leq i_{1}, \ldots, i_{2 k} \leq n} d\left(\Omega_{i_{1}}{ }^{i_{2}} \wedge \ldots \wedge \Omega_{i_{2 k-3}}{ }^{i_{2 k-2}} \wedge \omega_{i_{2 k-1}}{ }^{i_{2 k}} \wedge \theta^{[I]}\right) \\
& \quad-\sum_{1 \leq i_{1}, \ldots, i_{2 k} \leq n} d\left(\Omega_{i_{1}}{ }^{i_{2}} \wedge \ldots \wedge \Omega_{i_{2 k-3}}{ }^{i_{2 k-2}}\right) \wedge \omega_{i_{2 k-1}}{ }^{i_{2 k}} \wedge \theta^{[I]} \\
& +\sum_{1 \leq i_{1}, \ldots, i_{2 k} \leq n} \Omega_{i_{1}}{ }^{i_{2}} \wedge \ldots \wedge \Omega_{i_{2 k-3}}^{i_{2 k-2}} \wedge \omega_{i_{2 k-1}}^{i_{2 k}} \wedge d \theta^{[I]} \\
& -\sum_{1 \leq i_{1}, \ldots, i_{2 k} \leq n} \Omega_{i_{1}}{ }^{i_{2}} \wedge \ldots \wedge \Omega_{i_{2 k-3}}{ }^{i_{2 k-2}} \wedge \omega_{i_{2 k-1}} p_{k} \wedge \omega_{p_{k}}{ }^{i_{2 k}} \wedge \theta^{[I]} .
\end{aligned}
$$

Note that, by symmetry,

$$
\begin{aligned}
& \sum_{1 \leq i_{1}, \ldots, i_{2 k} \leq n} d \Omega_{i_{1}}{ }^{i_{2}} \wedge \Omega_{i_{3}}{ }^{i_{4}} \ldots \wedge \Omega_{i_{2 k-3}}{ }^{i_{2 k-2}} \wedge \omega_{i_{2 k-1}}{ }^{i_{2 k}} \wedge \theta^{[I]} \\
& \quad=\ldots=\sum_{1 \leq i_{1}, \ldots, i_{2 k} \leq n} \Omega_{i_{1}}^{{ }^{2}} \wedge \ldots \wedge \Omega_{i_{2 k-5}}{ }^{i_{2 k-4}} \wedge d \Omega_{i_{2 k-3}}{ }^{i_{2 k-2}} \wedge \omega_{i_{2 k-1}}{ }^{i_{2 k}} \wedge \theta^{[I]}
\end{aligned}
$$


which, by the second Bianchi identity and anti-symmetry, is equal to

$$
\begin{aligned}
& \sum_{1 \leq i_{1}, \ldots, i_{2 k} \leq n} \Omega_{i_{1}}{ }^{i_{2}} \wedge \ldots \wedge \Omega_{i_{2 k-5}}{ }^{i_{2 k-4}} \wedge \\
& \wedge\left(-\Omega_{i_{2 k-3}}{ }^{r_{k-1}} \wedge \omega_{r_{k-1}}{ }^{i_{2 k-2}}+\omega_{i_{2 k-3}}{ }^{r_{k-1}} \wedge \Omega_{r_{k-1}}{ }^{i_{2 k-2}}\right) \wedge \omega_{i_{2 k-1}}{ }^{i_{2 k}} \wedge \theta^{[I]} \\
&=-2 \sum_{1 \leq i_{1}, \ldots, i_{2 k} \leq n} \Omega_{i_{1}}{ }^{{ }^{2}} \wedge \ldots \wedge \Omega_{i_{2 k-5}}{ }^{i_{2 k-4}} \wedge \Omega_{i_{2 k-3}}{ }^{r_{k-1}} \wedge \omega_{r_{k-1}}{ }^{i_{2 k-2}} \wedge \omega_{i_{2 k-1}} i_{2 k} \wedge \theta^{[I]} .
\end{aligned}
$$

Next, we compute $d \theta^{[I]}$. Assume first that $I=\left(i_{1}, \ldots, i_{2 k}\right) \in S_{n, 2 k}$. We supplement $I$ with $i_{2 k+1}, \ldots, i_{n}$ so that $\left(i_{1}, \ldots, i_{n}\right)$ is a permutation of $(1, \ldots, n)$. We have

$$
\theta^{[I]}=\delta_{1 \ldots n}^{i_{1} \ldots i_{n}} \theta^{i_{2 k+1}} \wedge \ldots \wedge \theta^{i_{n}}
$$

In view of the first structural equations, this implies that

$$
\begin{aligned}
d \theta^{[I]} & =\delta_{1 \ldots n}^{i_{1} \ldots i_{n}} \sum_{s=2 k+1}^{n} \sum_{t=1}^{2 k}(-1)^{s-1} \theta^{i_{2 k+1}} \wedge \ldots \wedge \theta^{i_{s-1}} \wedge\left(\theta^{i_{t}} \wedge \omega_{i_{t}}{ }^{i_{s}}\right) \wedge \theta^{i_{s+1}} \wedge \ldots \wedge \theta^{i_{n}} \\
& =\delta_{1 \ldots n}^{i_{1} \ldots i_{n}} \sum_{t=1}^{2 k} \sum_{s=2 k+1}^{n}(-1)^{s} \omega_{i_{t}}{ }^{i_{s}} \wedge \theta^{i_{t}} \wedge \theta^{i_{2 k+1}} \wedge \ldots \wedge \theta^{i_{s-1}} \wedge \theta^{i_{s+1}} \wedge \ldots \wedge \theta^{i_{n}} \\
& =\sum_{t=1}^{2 k} \sum_{s=2 k+1}^{n} \omega_{i_{t}}{ }^{i_{s}} \wedge \theta^{\left[I: i_{t} \rightarrow i_{s}\right]} \\
& =\sum_{t=1}^{2 k} \sum_{s=1}^{n} \omega_{i_{t}}{ }^{s} \wedge \theta^{\left[I: i_{t} \rightarrow s\right]},
\end{aligned}
$$

where $I: i_{t} \rightarrow i_{s}$ denotes $\left(i_{1}, \ldots, i_{t-1}, i_{s}, i_{t+1}, \ldots, i_{2 k}\right)$. This continues to holds when for general multi-index $I=\left(i_{1}, \ldots, i_{2 k}\right) \in\{1, \ldots, n\}^{k}$. Indeed, if $I \notin S_{n, 2 k}$, we have $d \theta^{[I]}=0$ and

$$
\begin{aligned}
& \sum_{t=1}^{2 k} \sum_{s=1}^{n} \omega_{i_{t}}{ }^{s} \wedge \theta^{\left[I: i_{t} \rightarrow s\right]} \\
& \quad=\sum_{\text {special }} \sum_{t^{\prime} s}^{n} \omega_{s=1}{ }^{s} \wedge \theta^{\left[I: i_{t} \rightarrow s\right]} \\
& \quad=\frac{1}{2} \sum_{\text {special }} \sum_{t^{\prime} s}^{n}\left(\omega^{n}{ }^{s}{ }^{s} \wedge \theta^{\left[I: i_{t} \rightarrow s\right]}+\omega_{i_{\tilde{t}}}{ }^{s} \wedge \theta^{\left[I: i_{\tilde{t}} \rightarrow s\right]}\right) \\
& =0 .
\end{aligned}
$$


where the set of special $t$ 's are those such that there is a unique $\tilde{t} \neq t$ such that $i_{t}=i_{\tilde{t}}$. It thus follows that

$$
\begin{aligned}
& \sum_{1 \leq i_{1}, \ldots, i_{2 k} \leq n} \Omega_{i_{1}}{ }^{i_{2}} \wedge \ldots \wedge \Omega_{i_{2 k-3}}{ }^{i_{2 k-2}} \wedge \omega_{i_{2 k-1}}{ }^{i_{2 k}} \wedge d \theta^{[I]} \\
& =\sum_{1 \leq i_{1}, \ldots, i_{2 k} \leq n} \Omega_{i_{1}}{ }^{i_{2}} \wedge \ldots \wedge \Omega_{i_{2 k-3}}{ }^{i_{2 k-2}} \wedge \omega_{i_{2 k-1}}{ }^{i_{2 k}} \wedge \sum_{t=1}^{2 k} \sum_{s=1}^{n} \omega_{i_{t}}{ }^{s} \wedge \theta^{\left[I: i_{t} \rightarrow s\right]} \\
& =2(k-1) \sum_{1 \leq i_{1}, \ldots, i_{2 k}, s \leq n} \Omega_{i_{1}}^{i_{2}} \wedge \ldots \wedge \Omega_{i_{2 k-3}{ }^{i_{2 k-2}}} \wedge \omega_{i_{2 k-1}}{ }^{i_{2 k}} \wedge \omega_{i_{2 k-2}} s \wedge \theta^{\left[I: i_{2 k-2} \rightarrow s\right]} \\
& +2 \sum_{1 \leq i_{1}, \ldots, i_{2 k}, s \leq n} \Omega_{i_{1}}^{i_{2}} \wedge \ldots \wedge \Omega_{i_{2 k-3}}^{i_{2 k-2}} \wedge \omega_{i_{2 k-1}}^{i_{2 k}} \wedge \omega_{i_{2 k}}{ }^{s} \wedge \theta^{\left[I: i_{2 k} \rightarrow s\right]} \\
& =-2(k-1) \sum_{1 \leq j_{1}, \ldots, j_{2 k}, i_{2 k-2} \leq n} \Omega_{j_{1}}^{j_{2}} \wedge \ldots \wedge \Omega_{j_{2 k-3}}^{i_{2 k-2}} \wedge \omega_{i_{2 k-2}}^{j_{2 k-2}} \wedge \omega_{j_{2 k-1}}^{j_{2 k}} \wedge \theta^{[J]} \\
& +2 \sum_{1 \leq j_{1}, \ldots, j_{2 k}, i_{2 k} \leq n} \Omega_{j_{1}}^{{ }^{j}} \wedge \ldots \wedge \Omega_{j_{2 k-3}}^{j_{2 k-2}} \wedge \omega_{j_{2 k-1}}^{i_{2 k}} \wedge \omega_{i_{2 k}}{ }^{j_{2 k}} \wedge \theta^{[J]} .
\end{aligned}
$$

We thus get

$$
\begin{aligned}
2^{k} k ! \Lambda_{k}= & \sum_{1 \leq i_{1}, \ldots, i_{2 k} \leq n} d\left(\Omega_{i_{1}}{ }^{{ }_{2}} \wedge \ldots \wedge \Omega_{i_{2 k-3}}{ }^{i_{2 k-2}} \wedge \omega_{i_{2 k-1}}{ }^{i_{2 k}} \wedge \theta^{[I]}\right) \\
& +\sum_{1 \leq i_{1}, \ldots, i_{2 k}, p_{k} \leq n} \Omega_{i_{1}}{ }^{i_{2}} \wedge \ldots \wedge \Omega_{i_{2 k-3}}{ }^{i_{2 k-2}} \wedge \omega_{i_{2 k-1}}{ }^{p_{k}} \wedge \omega_{p_{k}}{ }^{i_{2 k}} \wedge \theta^{[I]}
\end{aligned}
$$

Set

$$
\begin{aligned}
& \Lambda_{k}^{1}=\frac{1}{2^{k} k !} \sum_{1 \leq i_{1}, \ldots, i_{2 k} \leq n} \Omega_{i_{1}}{ }^{i_{2}} \wedge \ldots \wedge \Omega_{i_{2 k-3}}{ }^{i_{2 k-2}} \wedge \omega_{i_{2 k-1}}{ }^{i_{2 k}} \wedge \theta^{[I]}, \\
& \Lambda_{k}^{2}=\frac{1}{2^{k} k !} \sum_{1 \leq i_{1}, \ldots, i_{2 k}, p_{k} \leq n} \Omega_{i_{1}}{ }^{i_{2}} \wedge \ldots \wedge \Omega_{i_{2 k-3}}{ }^{i_{2 k-2}} \wedge \omega_{i_{2 k-1}}{ }^{p_{k}} \wedge \omega_{p_{k}}{ }^{i_{2 k}} \wedge \theta^{[I]} .
\end{aligned}
$$

Then $\Lambda_{k}=d \Lambda_{k}^{1}+\Lambda_{k}^{2}$. It should be noted that, unlike $\Lambda_{k}, \Lambda_{k}^{1}$ and $\Lambda_{k}^{2}$ are frame dependent.

\section{$3 \quad$ Higher order mass}

Let $\left(M^{n}, g\right)$ be a Riemannian manifold and assume that there is a compact set $K \subset M$ such that $M \backslash K$ has an asymptotic flat structure of order $\tau$ : There are some $R \geq 1$ and a diffeomorphism $\Phi: M \backslash K \rightarrow \mathbb{R}^{n} \backslash B_{R}$ such that

$$
\left(\Phi^{*} g\right)_{i j}(x)=\delta_{i j}+o_{2}\left(|x|^{-\tau}\right) \text { as }|x| \rightarrow \infty,
$$


where $x=\left(x^{1}, \ldots, x^{n}\right)$ is the coordinate function with respect to $\Phi$ and we write $f=o_{l}\left(|x|^{-\tau}\right)$ if $\partial_{i_{1}} \ldots \partial_{i_{p}} f=o\left(|x|^{-\tau-p}\right)$ for any $1 \leq p \leq l$. We also assume that

$$
\Lambda_{k} \in L^{1}(M) \text {. }
$$

For simplicity, we only consider the case where $M$ has one end; the general case requires minor modification.

With respect to the asymptotic structure $\Phi$, let $e_{i}=\partial_{x^{i}}$ and $\theta^{i}=d x^{i}$. The connection one-forms and curvature two-forms are defined by

$$
\nabla_{X} e_{i}=\omega_{i}{ }^{j}(X) e_{j} \text { and } \Omega_{i}{ }^{j}(X, Y)=\theta^{j}\left(R(X, Y) e_{i}\right)
$$

We note that $\omega_{i}{ }^{j}$ and $\Omega_{i}{ }^{j}$ may not be skew-symmetric. Define the $(n-1)$-form

$$
\Lambda_{k}^{1}(\Phi)=\frac{1}{2^{k} k !} \sum_{1 \leq i_{1}, \ldots, i_{2 k} \leq n} \Omega_{i_{1}}^{i_{2}} \wedge \ldots \wedge \Omega_{i_{2 k-3}}{ }^{i_{2 k-2}} \wedge \omega_{i_{2 k-1}} i_{2 k} \wedge \theta^{[I]}
$$

and the associate " $k$-th order mass"

$$
m_{k}(\Phi)=\lim _{R \rightarrow \infty} \int_{S_{R}}(-1)^{k} \Lambda_{k}^{1}(\Phi)
$$

Here $S_{R}$ denotes the coordinate sphere of radius $R$ centered at the origin.

To show that $m_{k}(\Phi)$ is well-defined, we use the decomposition of $\Lambda_{k}$ which we derived earlier (in an orthonormal frame). We first use the Gram-Schmidt orthogonalization to construct an orthonormal frame:

$$
\begin{aligned}
& \tilde{e}_{1}=\frac{e_{1}}{\left|e_{1}\right|}, \\
& \tilde{e}_{2}=\frac{e_{2}-g\left(e_{2}, \tilde{e}_{1}\right) \tilde{e}_{1}}{\left|e_{2}-g\left(e_{2}, \tilde{e}_{1}\right) \tilde{e}_{1}\right|}, \\
& \ldots \\
& \tilde{e}_{n}=\frac{e_{n}-g\left(e_{n}, \tilde{e}_{1}\right) \tilde{e}_{1}-\ldots-g\left(e_{n}, \tilde{e}_{n-1}\right) \tilde{e}_{n-1}}{\left|e_{n}-g\left(e_{n}, \tilde{e}_{1}\right) \tilde{e}_{1}-\ldots-g\left(e_{n}, \tilde{e}_{n-1}\right) \tilde{e}_{n-1}\right|} .
\end{aligned}
$$

Evidently,

$$
\tilde{e}_{i}=e_{i}+o_{2}\left(|x|^{-\tau}\right) .
$$

Let $\left\{\tilde{\theta}^{i}\right\}$ be the dual frame to $\left\{\tilde{e}_{i}\right\}$ and define the connection one-forms $\tilde{\omega}_{i}^{j}$ and curvature two-forms $\tilde{\Omega}_{i}{ }^{j}$ accordingly. Set

$$
\begin{aligned}
& \tilde{\Lambda}_{k}^{1}=\frac{1}{2^{k} k !} \sum_{1 \leq i_{1}, \ldots, i_{2 k} \leq n} \tilde{\Omega}_{i_{1}}{ }^{i_{2}} \wedge \ldots \wedge \tilde{\Omega}_{i_{2 k-3}}{ }^{i_{2 k-2}} \wedge \tilde{\omega}_{i_{2 k-1}}{ }^{i_{2 k}} \wedge \tilde{\theta}^{[I]}, \\
& \tilde{\Lambda}_{k}^{2}=\frac{1}{2^{k} k !} \sum_{1 \leq i_{1}, \ldots, i_{2 k}, p_{k} \leq n} \tilde{\Omega}_{i_{1}}{ }^{i_{2}} \wedge \ldots \wedge \tilde{\Omega}_{i_{2 k-3}}{ }^{i_{2 k-2}} \wedge \tilde{\omega}_{i_{2 k-1}}{ }^{p_{k}} \wedge \tilde{\omega}_{p_{k}}{ }^{i_{2 k}} \wedge \tilde{\theta}^{[I]} .
\end{aligned}
$$


Then $\Lambda_{k}=d \tilde{\Lambda}_{k}^{1}+\tilde{\Lambda}_{k}^{2}$.

To relate $\tilde{\omega}_{i}{ }^{j}$ and $\tilde{\Omega}_{i}{ }^{j}$ to $\omega_{i}{ }^{j}$ and $\Omega_{i}{ }^{j}$, we write

$$
\tilde{e}_{i}=P_{i}^{j} e_{j} \text { and } \tilde{\theta}^{j}=\left(P^{t}\right)_{i}{ }^{j} \theta^{i}
$$

where the matrix $P$ satisfies

$$
P_{i}^{j}=\delta_{i j}+o_{2}\left(|x|^{-\tau}\right)
$$

We have

$$
\nabla_{X} \tilde{e}_{i}=\nabla_{X}\left(P_{i}^{j} e_{j}\right)=\left(d P_{i}^{j}(X)+P_{i}^{k} \omega_{k}^{j}(X)\right) e_{j}=\left(d P_{i}^{j}(X)+P_{i}^{k} \omega_{k}^{j}(X)\right)\left(P^{-1}\right)_{j}{ }^{l} \tilde{e}_{l},
$$

which implies that

$$
\tilde{\omega}_{i}{ }^{j}=\omega_{i}{ }^{j}+d P_{i}^{j}+o_{1}\left(|x|^{-2 \tau-1}\right)
$$

Likewise

$$
\tilde{\Omega}_{i}{ }^{j}=\tilde{\theta}_{j}\left(R(X, Y) \tilde{e}_{i}\right)=\left(P^{t}\right)_{j}{ }^{k} P_{i}^{l} \theta_{k}\left(R(X, Y) e_{l}\right)=\left(P^{t}\right)_{j}{ }^{k} P_{i}{ }^{l} \Omega_{k}{ }^{l}=\Omega_{i}{ }^{j}+o\left(|x|^{-2 \tau-2}\right) .
$$

From the above computation, we see that

$$
\tilde{\Lambda}_{k}^{2}=O\left(|x|^{-(\tau(k+1)+2 k)}\right) \in L^{1}(M),
$$

and

$$
\begin{aligned}
\tilde{\Lambda}_{k}^{1}-\Lambda_{k}^{1}=d & \left(\frac{1}{2^{k} k !} \sum_{1 \leq i_{1}, \ldots, i_{2 k} \leq n}\left[P_{i_{2 k-1}} i_{2 k}-\delta_{i_{2 k-1} i_{2 k}}\right] \Omega_{i_{1}}{ }^{i_{2}} \wedge \ldots \wedge \Omega_{i_{2 k-3}} i_{2 k-2} \wedge \theta^{[I]}\right) \\
& +o\left(|x|^{-(\tau(k+1)+2 k)+1}\right) .
\end{aligned}
$$

These lead to, for $R \gg N \gg R_{0}$,

$$
\begin{aligned}
\int_{S_{R}} \Lambda_{k}^{1} & =\int_{S_{R}} \tilde{\Lambda}_{k}^{1}+o\left(R^{n-(\tau(k+1)+2 k)}\right) \\
& =\int_{S_{N}} \tilde{\Lambda}_{k}^{1}+\int_{B_{R} \backslash B_{N}}\left[\Lambda_{k}+\tilde{\Lambda}_{k}^{2}\right]+o\left(R^{n-(\tau(k+1)+2 k)}\right) .
\end{aligned}
$$

Hence, for

$$
\tau>\frac{n-2 k}{k+1}
$$

the mass $m_{k}(\Phi)$ is well-defined.

In fact, the argument above shows that if $D_{j}$ is an exhaustion of $M$ by closed sets such that

$$
R_{j}=\inf \left\{|x|: x \in \partial D_{k}\right\} \rightarrow \infty \text { and } R_{j}^{-(n-1)}\left|\partial D_{k}\right| \text { remains bounded as } j \rightarrow \infty
$$


then

$$
m_{k}(\Phi)=\lim _{j \rightarrow \infty} \int_{S_{j}}(-1)^{k} \Lambda_{k}^{1} .
$$

We show next that $m_{k}(\Phi)$ is independent of the asymptotic structure $\Phi$. Assume that $\tilde{\Phi}$ is another asymptotic structure of $(M, g)$ and let $\tilde{x}=\left(\tilde{x}^{1}, \ldots, \tilde{x}^{n}\right)$ denotes the coordinate function with respect to $\tilde{\Phi}$. To show that $m_{k}(\Phi)=m_{k}(\tilde{\Phi})$ we appeal to a theorem of Bartnik [2, Theorem 3.1] to find harmonic coordinates $y=\left(y^{1}, \ldots, y^{n}\right)$ and $\tilde{y}=\left(\tilde{y}^{1}, \ldots, \tilde{y}^{n}\right)$ such that

$$
\begin{aligned}
& \left|x^{i}-y^{i}\right|+\left|\tilde{x}^{i}-\tilde{y}^{i}\right|=o\left(|x|^{1-\tau}\right)=o\left(|\tilde{x}|^{1-\tau}\right), \\
& \left|g\left(\partial_{x^{i}}, \partial_{x^{j}}\right)-g\left(\partial_{y^{i}}, \partial_{y^{j}}\right)\right|+\left|g\left(\partial_{\tilde{x}^{i}}, \partial_{\tilde{x}^{j}}\right)-g\left(\partial_{\tilde{y}^{i}}, \partial_{\tilde{y}^{j}}\right)\right|=o\left(|x|^{-\tau}\right)=o\left(|\tilde{x}|^{-\tau}\right), \\
& y^{i}=A_{j}{ }^{i} \tilde{y}^{j}+c^{i}
\end{aligned}
$$

where $A_{j}{ }^{i}$ and $c^{i}$ are constants. Note that the second relation and that the metric $g$ is asymptotically flat implies that the matrix $A=\left(A_{j}{ }^{i}\right)$ is orthonormal. Now the argument above show that in defining $m_{k}(\Phi)$ we can use the coordinate functions $y^{i}$ 's instead of the $x^{i}$ 's. Likewise $m_{k}(\tilde{\Phi})$ can be computed using $\tilde{y}^{i}$ 's instead of $\tilde{x}^{i}$ 's. But the frame $\partial_{y^{i}}$ and $\partial_{\tilde{y}^{i}}$ differ from one another by a rigid rotation: $\partial_{y_{i}}=A_{i}{ }^{j} \partial_{\tilde{y}^{j}}$. The argument proving that $\Lambda_{k}$ is frame independent applies showing that $\Lambda_{k}^{1}\left(\partial_{y^{i}}\right)=$ $\Lambda_{k}^{1}\left(\partial_{\tilde{y}^{i}}\right)$. This proves that $m_{k}=m_{k}(\Phi)$ is independent of $\Phi$.

We have thus shown that

Theorem 3.1 Let $(M, g)$ be a complete $n$-dimensional Riemannian manifold which is asymptotically flat of order $\tau$, i.e. there is a compact set $K$ and a diffeomorphism $\Phi: M \backslash K \rightarrow \mathbb{R}^{n} \backslash B_{R_{0}}$ for some $R_{0}>0$ such that in such coordinate system the metric $g$ satisfies $g_{i j}=\delta_{i j}+o_{3}\left(|x|^{-\tau}\right)$. Assume further that the curvature $\Lambda_{k}$ belongs to $L^{1}(M)$. Fix $1 \leq k<\frac{n}{2}$. If $\tau>\frac{n-2 k}{k+1}$, then the mass

$$
m_{k}=\lim _{R \rightarrow \infty} \int_{S_{R}}(-1)^{k} \Lambda_{k}^{1}
$$

is well-defined and is independent of the asymptotic structure at infinity.

To finish this section, we give an example. Fix some $1 \leq k<\frac{n}{2}$. Consider an asymptotically flat manifold where the metric takes the following form at infinity

$$
g_{i j}=\exp \left(\frac{2 m}{r^{\frac{n-2 k}{k}}}\right) \delta_{i j}+o_{2}\left(r^{-\frac{n-2 k}{k}}\right) .
$$


The connection one-forms and the curvature two-forms are

$$
\begin{aligned}
\omega_{i}^{j} & =\Gamma_{i k}^{j} d x^{k}=-\frac{(n-2 k)}{k} \frac{m}{r^{\frac{n}{k}}}\left(\delta_{i j} r d r+x^{i} d x^{j}-x^{j} d x^{i}\right)+o\left(r^{1-\frac{n}{k}}\right), \\
\Omega_{i}{ }^{j} & =d \omega_{i}^{j}-\omega_{i}{ }^{i} \wedge \omega_{t}{ }^{j} \\
& =\frac{(n-2 k) n}{k^{2}} \frac{m}{r^{\frac{n+k}{k}}} d r \wedge\left(x^{i} d x^{j}-x^{j} d x^{i}\right)-\frac{2(n-2 k)}{k} \frac{m}{r^{\frac{n}{k}}} d x^{i} \wedge d x^{j}+o\left(r^{-\frac{n}{k}}\right) .
\end{aligned}
$$

Thus,

$$
\left.\Lambda_{k}^{1}\right\rfloor S_{R}=c(n, k) \frac{(-1)^{k} m^{k}}{R^{n}} \sum_{1 \leq i, j \leq n}\left(x^{i} d x^{j}-x^{j} d x^{i}\right) \wedge *\left(d x^{i} \wedge d x^{j}\right)
$$

and so

$$
m_{k}=c(n, k) m^{k}
$$

where $c(n, k)$ is some positive constant.

The above computation also shows that, for the time symmetric slice of the Schwarzschild spacetime in higher dimensions, only the first mass (which is the same as the ADM mass) is nonzero. All the higher order masses, if well-defined, vanish.

\section{On the non-negativity of the $k$-th mass}

It is of interest to see if the $k$-th mass is non-negative under some assumption on either the $\Lambda_{k}$ or the $\sigma_{k}$ curvature. We are only able to do so under a very restrictive hypothesis that $(M, g)$ is locally conformally flat and that $\lambda\left(A_{g}\right)$ is asymptotically on the boundary of the $\Gamma_{k}$ cone. Of the two assumptions, we believe the local conformal flatness assumption is more severe. Note also that, in Schoen and Yau's proof of the positive mass theorem [22, 23], one can assume without loss of generality that the manifold is asymptotically scalar flat (i.e. $\left.\lambda\left(A_{g}\right) \in \partial \Gamma_{1}\right)$.

Theorem 4.1 Let $(M, g)$ be a complete, asymptotically flat Riemannian manifold of dimension $n \geq 3$ and let $2 \leq k<\frac{n}{2}$. If, near a given end, $g$ is locally conformally flat, $A_{g}$ belongs to the $\bar{\Gamma}_{k}$ cone and the $\Lambda_{k}$ curvature vanishes, then the $k$-th mass of that end is non-negative. Furthermore, if the $k$-th mass is zero, then, near that end, $(M, g)$ is isometric to an Euclidean end.

The proof of this theorem has a different flavor from what is presented in this paper and will be published elsewhere. 


\section{References}

[1] Richard L. Arnowitt, Stanley Deser, and Charles W. Misner, Coordinate invariance and energy expressions in general relativity, Phys.Rev. 122, 997.

[2] Robert Bartnik, The mass of an asymptotically flat manifold, Comm. Pure Appl. Math. 39 (1986), no. 5, 661-693.

[3] Sun-Yung A. Chang, Matthew J. Gursky, and Paul Yang, An equation of MongeAmpère type in conformal geometry, and four-manifolds of positive Ricci curvature, Ann. of Math. (2) 155 (2002), no. 3, 709-787.

[4] - An a priori estimate for a fully nonlinear equation on four-manifolds, J. Anal. Math. 87 (2002), 151-186, Dedicated to the memory of Thomas H. Wolff.

[5] _ A conformally invariant sphere theorem in four dimensions, Publ. Math. Inst. Hautes Études Sci. (2003), no. 98, 105-143.

[6] _ Entire solutions of a fully nonlinear equation, Lectures on partial differential equations, New Stud. Adv. Math., vol. 2, Int. Press, Somerville, MA, 2003, pp. 43-60.

[7] Sun-Yung Alice Chang and Hao Fang, A class of variational functionals in conformal geometry, Int. Math. Res. Not. IMRN (2008), no. 7, Art. ID rnn008, 16.

[8] Olivier Druet, Compactness for Yamabe metrics in low dimensions, Int. Math. Res. Not. (2004), no. 23, 1143-1191.

[9] Yuxin Ge and Guofang Wang, On a fully nonlinear Yamabe problem, Ann. Sci. École Norm. Sup. (4) 39 (2006), no. 4, 569-598.

[10] C. Robin Graham, Extended obstruction tensors and renormalized volume coefficients, Adv. Math. 220 (2009), no. 6, 1956-1985.

[11] Pengfei Guan and Guofang Wang, A fully nonlinear conformal flow on locally conformally flat manifolds, J. Reine Angew. Math. 557 (2003), 219-238.

[12] Matthew J. Gursky and Jeff A. Viaclovsky, Prescribing symmetric functions of the eigenvalues of the Ricci tensor, Ann. of Math. (2) 166 (2007), no. 2, 475-531.

[13] M. A. Khuri, F. C. Marques, and R. M. Schoen, A compactness theorem for the Yamabe problem, J. Differential Geom. 81 (2009), no. 1, 143-196. 
[14] Aobing Li and YanYan Li, On some conformally invariant fully nonlinear equations, Comm. Pure Appl. Math. 56 (2003), no. 10, 1416-1464.

[15] _ On some conformally invariant fully nonlinear equations. II. Liouville, Harnack and Yamabe, Acta Math. 195 (2005), 117-154.

[16] YanYan Li and Luc Nguyen, A fully nonlinear version of the Yamabe problem on locally conformally flat manifolds with umbilic boundary, (2009), http://arxiv.org/abs/0911.3366v1.

[17] YanYan Li and Lei Zhang, Compactness of solutions to the Yamabe problem. II, Calc. Var. Partial Differential Equations 24 (2005), no. 2, 185-237.

[18] Compactness of solutions to the Yamabe problem. III, J. Funct. Anal. 245 (2007), no. 2, 438-474.

[19] YanYan Li and Meijun Zhu, Yamabe type equations on three-dimensional Riemannian manifolds, Commun. Contemp. Math. 1 (1999), no. 1, 1-50.

[20] Fernando Coda Marques, A priori estimates for the Yamabe problem in the nonlocally conformally flat case, J. Differential Geom. 71 (2005), no. 2, 315-346.

[21] Richard Schoen, Conformal deformation of a Riemannian metric to constant scalar curvature, J. Differential Geom. 20 (1984), no. 2, 479-495.

[22] Richard Schoen and Shing Tung Yau, On the proof of the positive mass conjecture in general relativity, Comm. Math. Phys. 65 (1979), no. 1, 45-76.

[23] _ Proof of the positive mass theorem. II, Comm. Math. Phys. 79 (1981), no. 2, 231-260. MR 612249 (83i:83045)

[24] Richard M. Schoen, On the number of constant scalar curvature metrics in a conformal class, Differential geometry, Pitman Monogr. Surveys Pure Appl. Math., vol. 52, Longman Sci. Tech., Harlow, 1991, pp. 311-320.

[25] Wei-Min Sheng, Neil S. Trudinger, and Xu-Jia Wang, The Yamabe problem for higher order curvatures, J. Differential Geom. 77 (2007), no. 3, 515-553.

[26] Neil S. Trudinger and Xu-Jia Wang, On Harnack inequalities and singularities of admissible metrics in the Yamabe problem, Calc. Var. Partial Differential Equations 35 (2009), no. 3, 317-338.

[27] - The intermediate case of the Yamabe problem for higher order curvatures, Int. Math. Res. Not. IMRN (2010), no. 13, 2437-2458. 
[28] Jeff A. Viaclovsky, Conformal geometry, contact geometry, and the calculus of variations, Duke Math. J. 101 (2000), no. 2, 283-316.

[29] _ Conformally invariant Monge-Ampère equations: global solutions, Trans. Amer. Math. Soc. 352 (2000), no. 9, 4371-4379.

[30] __ Estimates and existence results for some fully nonlinear elliptic equations on Riemannian manifolds, Comm. Anal. Geom. 10 (2002), no. 4, 815-846. 